\title{
Quorum Sensing Inhibitory Potential and Molecular Docking Studies of Phyllanthus Emblica Phytochemicals Against Pseudomonas Aeruginosae
}

Sharmila Baburam

Muthayammal College of Arts \& Science Rasipuram

Srinivasan Ramasamy

Tamil Nadu State Council for Science \& Technology

Gnanendra Shanmugam

Yeungnam University

Maghimaa Mathanmohun ( $\square$ mmaghimaa@gmail.com )

Muthayammal College of Arts \& Science Rasipuram https://orcid.org/0000-0002-9043-435X

\section{Research Article}

Keywords: quorum sensing, Phyllanthus emblica, Pseudomonas aeruginosa, molecular docking

Posted Date: August 2nd, 2021

DOl: https://doi.org/10.21203/rs.3.rs-663878/v1

License: (1) (1) This work is licensed under a Creative Commons Attribution 4.0 International License.

Read Full License 


\section{Abstract}

Phyllanthus emblica a traditional medicinal plant that is endowed with curative properties including antibacterial, anti-fungal, anti-viral, and analgesic properties. Bacteria make use of cell-cell signaling system known as Quorum sensing (QS) and respond to their own population. In most gram-negative bacteria, the transcriptional regulators belonging to the Lux R protein play a crucial role in the QS mechanism by detecting the presence of signaling molecules known as $\mathrm{N}$-acyl homoserine lactones (AHLs). In this present work, the anti-quorum sensing activity of Phyllanthus emblica was evaluated against Pseudomonas aeruginosa. Anti-quorum sensing efficacy of Phyllanthus emblica was estimated with reference to QS Bio-monitoring strain Chromobacterium violaceum. The binding efficacy of the phytochemicals of Phyllanthus emblica against CviR Protein from Chromobacterium violaceum and LasR Protein from Phyllanthus emblica were studied.

\section{Introduction}

Globally, in the last few decades the emergence and widespread of antimicrobial-resistant, antimicrobial drug-resistant strains of Pseudomonas spp. and Staphylococcus spp. become the alarming situation of greater public health concern [1]. Pseudomonas aeruginosa is mainly responsible for postoperative wound infections, and is more prevalent in most of the hospital-acquired infections [2].

In general, antibiotics are used to control these microbial infections by inhibiting their growth. However, the continuous usage and misuse of antibiotic therapy led to the emergence of multi-drug resistant strains to the tolerance against a broad spectrum of available antibiotics [3]. The development of these multiple drug-resistant bacteria has forced the scientists to search for new antibacterial agents have become the main concern [4]. Though the search for new antimicrobial substances has resulted in novel antimicrobial chemotherapeutic agents as synthetic drugs from various sources, the higher cost production and its adverse effects has limited its usages when compared to plant-derived drugs [5]. Thus the search for novel anti-pathogenic agents has increased the focus on the potential compounds from plant sources that are widespread across the globe. The increase in the search for therapeutic compounds from plants is based on a fact that plants continue to survive with high bacterial density in an environment and might possess protective means against infections. Thus in recent years, the extracts from plants and the knowledge of medicinal plants has gained the attentions of many pharmaceutical industries [6].

Cell-cell signaling systems known as Quorum sensing (QS) are used by bacteria to communicate with each other and respond to their own population. In gram-negative bacteria, the LuxR, a transcriptional regulator protein plays a central role in the QS mechanism to detect $\mathrm{N}$-acyl-homoserine lactones (AHLs) as signalling molecules [7-8]

In this work, the anti-quorum sensing activity of Phyllanthus emblica was evaluated against Pseudomonas aeruginosa. Even though the majority of the isolates were sensitive to most of the 
antibiotics, the lactamase which means that they quickly become resistant to standard antibiotics during treatment, requiring a change in antibiotic to avoid worsening of the sepsis [9]. Anti-quorum sensing efficacy of Phyllanthus emblica was estimated with reference to QS bio-monitoring strain Chromobacterium violaceum. The binding efficacy of the phytochemicals against CviR Protein from Chromobacterium violaceum and LasR Protein from Pseudomonas aeruginosa were studied.

\section{Methodology}

The 3D models of Pseudomonas aeruginosa LasR (PDB ID: 2UV0) and CviR from Chromobacterium violaceum (PDB ID: 3QP5) were retrieved from the PDB database and the conserved residues were determined with other LuxR family protein by using ClustalW at the EBI server [9].

\section{Ligands}

The principle compounds of Phyllanthus emblica was retrieved from Duke Ethano botanical database and their respective structures were obtained from Pubchem Database. The structures were retrieved in SDF format.

\section{Docking studies}

The retrieved compounds in SDF file format from Pubchem database were docked with the amino acids in the binding site of CviR and LasR using the default parameters. The interactions of principle compounds with LasR in the docked complex were analyzed by the pose-view of LeadIT [10]. Pose-view tools [11] were used to study the interactions of compounds with CviR and LasR in the docked complex.

\section{Results}

The 3D models of Pseudomonas aeruginosa LasR (PDB ID: 2UV0) and CviR from Chromobacterium violaceum (PDB ID: 3QP5) retrieved from PDB database were shown in Fig. 1. The homologies between the proteins belonging to the LuxR family (quorum sensing enhanced transcriptional regulators) were analyzed by multiple sequence alignment (Fig. 2). The binding site were determined by using the cocrystallized structures.

\section{Docking}

The Docking program FlexX, from LeadIT was used to dock Phyllanthus emblica compounds with the binding pocket of the LasR, CviR, and the developed model, LasR. The docking was carried out with a radius of $6.5 \mathrm{~A}^{0}$ at the site of docking.

\section{Docking Analysis}

The interactions between the binding site residues of CviR and the modeled protein LasR with the compounds as ligand molecules in the docked complexes were given in Table 1. A keen observation of 
these interacting residues of the LuxR family proteins, the modeled LasR, and the ligand molecules revealed the most important functional groups of the ligand molecules and the amino acids LuxR family proteins favoring the interactions (Table 2). The best-docked ligand molecules and their interactions with the amino acids in the active site of CviR and the modeled protein LasR is given in Figs. 3 and 4 .

Table 1

Docking Interactions of plant compounds with the active site amino acids of CviR from Chromobacterium Violaceum and their binding scores.

\begin{tabular}{|c|c|c|c|c|}
\hline \multirow[t]{2}{*}{ S.No. } & \multirow{2}{*}{$\begin{array}{l}\text { Compound } \\
\text { ID }\end{array}$} & \multicolumn{2}{|c|}{ Type of Interaction with active site residues } & \multirow{2}{*}{$\begin{array}{l}\text { Docking Score } \\
\text { (kJ/mol) }\end{array}$} \\
\hline & & Bonded & Non bonded & \\
\hline 1 & 57124935 & Asp86,Trp84 & Trp84,Asp86,Leu85 & -2.8686 \\
\hline 2 & 6437979 & - & Pro98,Asp97,Trp84,Tyr88 & -0.4569 \\
\hline 3 & 5481240 & Trp84,Asp97 & Trp84,Ile99.Asp97,Leu100,Tyr88 & -8.2647 \\
\hline 4 & 5366074 & Trp84,Leu85,Asp86 & Asp86,Leu85 & -8.3853 \\
\hline 5 & 5281126 & Trp84,Leu85,Asp86 & Leu85,Tyr88,Trp84,Leu100 & -0.0456 \\
\hline 6 & 5280934 & Leu85,Trp84,Asp86 & Trp84,Asp86,Tyr88,Leu85 & -1.4330 \\
\hline 7 & 5280442 & Gln87,Asp97 & Gln87,Tyr88,Trp84,Leu100,Asp97 & -9.7346 \\
\hline 8 & 641785 & Asp97,Tyr88 & Asp97,GIn87,Tyr88,Leu100 & -12.1467 \\
\hline 9 & 444539 & Trp84,Leu85,Asp86 & Trp84,Leu85 & -11.5130 \\
\hline 10 & 348962 & - & Asp97,Trp84,Tyr88,Pro98 & -4.5239 \\
\hline 11 & 301798 & Asp97,GIn87 & GIn87,Tyr88,Pro98,Trp84,Asp97 & -7.7922 \\
\hline 12 & 10465 & Asp86,Trp84,Leu85 & Leu85,Tyr88,Asp86 & -4.4680 \\
\hline 13 & 10416 & Trp84,Asp86,Leu85 & Leu85,Asp86 & -3.4816 \\
\hline 14 & 10212 & Trp84 & Trp84,Asp97,Tyr88,Ile99 & -6.9059 \\
\hline 15 & 1135 & Trp84,Leu85,Asp86 & Leu85 & -8.0549 \\
\hline 16 & 985 & Asp86,Leu85,Trp84 & Leu85,Asp86,Tyr88 & -3.1656 \\
\hline 17 & 323 & Asp86,Trp84,Leu85 & Trp84,Leu85 & -8.6772 \\
\hline $\begin{array}{l}\text { Refere } \\
\text { 3-oxo- }\end{array}$ & $\begin{array}{l}\text { ce Ligand } \\
6-\mathrm{HSL}\end{array}$ & Asp 97, Trp 84 & Tyr 88, Trp 84, Asp 97 & -8.3776 \\
\hline
\end{tabular}


Table 2

Docking Interactions of plant compounds with the active site amino acids of LasR from Pseudomonas aeruginosa and their binding scores.

\begin{tabular}{|c|c|c|c|c|}
\hline \multirow[t]{2}{*}{ S.No. } & \multirow{2}{*}{$\begin{array}{l}\text { Compound } \\
\text { ID }\end{array}$} & \multicolumn{2}{|c|}{ Type of Interaction with active site residues } & \multirow{2}{*}{$\begin{array}{l}\text { Docking Score } \\
\text { (kJ/mol) }\end{array}$} \\
\hline & & Bonded & Non bonded & \\
\hline 1 & 57124935 & Trp67 & Tyr71,Val82,Asp80,Tyr63,Trp67 & -2.6933 \\
\hline 2 & 6437979 & Asp80 & Tyr63,Asp80,Trp67,Tyr71 & -1.2080 \\
\hline 3 & 5481240 & Asp80,Trp67 & Trp67,Tyr63,Tyr71,Val82,Asp80 & -13.5553 \\
\hline 4 & 5366074 & Trp67 & Trp67,Val82,Tyr71,Tyr63 & -7.9594 \\
\hline 5 & 5281126 & Trp67 & Trp67,Tyr63,Tyr70 & -4.1555 \\
\hline 6 & 5280934 & Trp67 & Tyr70,Asp80,Tyr71,Trp67 & -5.1023 \\
\hline 7 & 5280442 & Trp67 & Trp67,Val82,Tyr63,Tyr71 & -12.9562 \\
\hline 8 & 641785 & Trp67,Asp80 & Asp80,Val82,Trp67,Tyr71,Tyr63 & -14.8740 \\
\hline 9 & 444539 & Asp80 & Tyr71,Tyr70,Trp67 & -9.3555 \\
\hline 10 & 348962 & Trp67 & Tyr63,Tyr71,Val82,Trp67,Asp80 & -9.6899 \\
\hline 11 & 301798 & Asp80 & Tyr63,Tyr71,Trp67 & -9.9831 \\
\hline 12 & 10465 & Trp67 & Tyr63,Tyr70,Trp67 & -7.7286 \\
\hline 13 & 10416 & Trp67 & Tyr71,Asp80,Tyr70,Trp67 & -7.3933 \\
\hline 14 & 10212 & Trp67 & Tyr71,Val82,Tyr63,Trp67 & -13.2575 \\
\hline 15 & 1135 & Asp80,Tyr63 & Trp67 & -8.3390 \\
\hline 16 & 985 & - & Tyr63,Tyr71,Val82,Trp67 & -8.1672 \\
\hline 17 & 323 & Trp67 & Trp67,Tyr71,Val82,Tyr63 & -9.7149 \\
\hline \multicolumn{2}{|c|}{$\begin{array}{l}\text { Reference Ligand } \\
\text { 3-oxo-octanoic acid }\end{array}$} & $\begin{array}{l}\text { Trp 67, Asp } \\
80\end{array}$ & Tyr 71, Tyr 63, Trp 67, Asp 80 & -8.3989 \\
\hline
\end{tabular}

\section{Discussion}

The Luxl homologs in most of the gram-negative bacteria generate the signal molecules, AHL. Usually, these signals were detected by the LuxR homologs present in them. Whereas in Pseudomonas aeruginosa the Luxl homolog is not been found, which makes the organism not to generate the signals of their own. Hence these bacteria cannot sense the signals from the same species. Instead, it responds to the signals produced by the other pathogenic bacteria. However, it encodes a LuxR homolog, LasR which 
can sense the signal molecules produced by the mixed community genera $[12,13]$. Thus LasR a transcriptional regulator was considered as a potential drug target.

The 3D structure of the target protein LasR from Pseudomonas aeruginosa was not available in any of the structural database, it was developed by using homology modeling method. The most homologous sequence in the Protein Data Bank was searched by using the BLASTP program. The BLASTP results showed that the Pseudomonas aeruginosa transcriptional regulator LasR is homologous with the structure CviR, LuxR- type transcriptional factor from Chromobacterium violaceum (PDB ID: 3QP5) over $40 \%$. As all these sequences belong to the same family, the structure of 3QP5 was considered as a template structure for comparative modeling. The model was generated by using Swiss model webserver.

The multiple sequence alignment (Fig. 2) of LuxR family proteins LasR from Pseudomonas aeruginosa, CviR from Chromobacterium violaceum, and LasR from Escherichia coli, Pseudomonas aeruginosa, and Enterobacter aerogens showed that amino acids are conserved in LuxR family proteins. These alignments enlighten that LasR from Pseudomonas aeruginosa is almost conserved. Hence, the structure of LasR from Pseudomonas aeruginosa was considered for further docking studies.

\section{Docking studies}

A total of 19 compounds were found as the principle compounds of the Euphorbia hirta. The 3D structures of these compounds were retrieved as SD files from the Pubchem database and were docked with the amino acids in the binding site of CviR from Chromobacterium violaceum and LasR from Pseudomonas aeruginosa by using FlexX. Out of these 19 compounds, 17 compounds formed docking complex with all both CviR and LasR and its binding energies were analyzed by LeadIT (Table 1 and 2). Considering the binding energy score, the 3 best-docked compounds for each protein CviR and LasR were selected (Figs. 3 and 4) and their docking interaction with the active site residues were analyzed by using the pose view of LeadIT.

The binding interactions in the docking studies of Chromobacterium violaceum CviR and Pseudomonas aeruginosa LasR with the 3 best-docked compounds of the Phyllanthus emblica exposed the similar binding of AHL residues, that are responsible for Quorum sensing activity. This result indicates that in Chromobacterium violaceum CviR, it is found that Tryptophan (Trp84) and Aspartic acid (Asp86 \& Asp97) plays a crucial role in exhibiting stronger interactions with ligands and these interactions were further supported by means of hydrophobic interactions by the contribution of Tyrosine (Try88). Similarly in Pseudomonas aeruginosa LasR, it is observed that Tryptophan (Trp67) and Aspartic acid (Asp80) are responsible for the bonded interactions with the ligands, and the non-bonded interaction, hydrophobic is facilitated by Tyrosine (Tyr 71 and Tyr 63).

The compounds CID_641785 (Cardamonin), CID_444539 (Cinnamic acid), and CID_5280442 (acacetin) exhibited the best docking scoring of $-12.1467 \mathrm{~kJ} / \mathrm{mol},-11.5130 \mathrm{~kJ} / \mathrm{mol}$, and $-9.7346 \mathrm{~kJ} / \mathrm{mol}$ respectively within the active site of $\mathrm{CviR}$ transcriptional regulator from Chromobacterium violaceum. It is observed that natural ligand 3-oxo-C6-HSL exhibited the docking score of $-8.3776 \mathrm{~kJ} / \mathrm{mol}$. Thus among 
the docked compounds, it is revealed that the compound of all the three compounds CID_641785, CID_444539, and CID_5280442 is having the highest docking score when compared to that of the natural ligand. Thus these compounds can be used to inhibit the quorum sensing mechanism in Chromobacterium violaceum.

The compounds CID_641785 (Cardamonin), CID_5481240 (Retusin), and CID_10212 (Imperatorin) exhibited the best docking scoring of $-14.8740 \mathrm{~kJ} / \mathrm{mol},-13.5553 \mathrm{~kJ} / \mathrm{mol}$, and $-13.2575 \mathrm{~kJ} / \mathrm{mol}$ respectively within the active site of LasR transcriptional regulator from Pseudomonas aeruginosa. It is observed that natural ligand 3-oxo-octanoic acid exhibited a docking score of $-8.3989 \mathrm{~kJ} / \mathrm{mol}$. Thus among the docked compounds, it is revealed that the compound CID_641785 is having the highest docking score when compared to that of the natural ligand. Thus this compound can be used to inhibit the quorum sensing mechanism in Pseudomonas aeruginosa.

The overall docking results of principle compounds with CviR and LasR proteins disclose the importance of the interacting amino acids Tryptophan, Aspartic acid, and Tyrosine (Y71). The docking studies revealed the necessary crucial hydrogen bond interactions with the critical amino acids and that of the compound Cardamonin (CID_641785) from Euphorbia hirta, with the highest binding score and might have a better inhibition activity against the quorum-sensing regulation of Pseudomonas aeruginosa.

\section{Conclusion}

Pseudomonas aeruginosa, an opportunistic pathogenic bacterium causing nosocomial infections, has quickly become resistant to standard antibiotics. The ability of antibiotics resistance is due to the effective communication among the bacterial cell. This communication is enhanced by transcriptional regulators belonging to LuxR protein that plays a crucial role in the QS mechanism by detecting the presence of signaling molecules known as $\mathrm{N}$-acylhomoserine lactones (AHLs) and regulates the pathogenicity. Pseudomonas aeruginosa harbors a transcriptional regulator LasR (Suppressor of cell division inhibition), that can recognize the AHLs to enhance the pathogenicity. Hence, LasR from Pseudomonas aeruginosa is considered as a valid drug target. Thus in the present study, the anti-quorum sensing activity of Phyllanthus emblica was evaluated against Pseudomonas aeruginosa. Anti-quorum sensing efficacy of Phyllanthus emblica was estimated with reference to QS Bio-monitoring strain Chromobacterium violaceum. The binding efficacy of the phytochemicals of Phyllanthus emblica was docked with the LasR from Pseudomonas aeruginosa and also with CviR Protein from Chromobacterium violaceum. This work discloses that amino acids Tryptophan, Aspartic acid, and Tyrosine (Y71) were important for the interactions. The docking studies also revealed the necessary crucial hydrogen bond interactions with the critical amino acids and that of the compound Cardamonin (CID_641785) with the highest binding score might be an effective inhibitor of Pseudomonas aeruginosa pathogenesis.

\section{Declarations}

\section{Acknowledgment}


The authors are thankful for the financial support from Tamilnadu State Council for Science \& Technology (TNSCST), DOTE Campus, Chennai (Science \& Technology Project: TNSCST/STPPRG/AR/2018-2019), and DST-FIST Centralized laboratory, Muthayammal College of Arts \& Science, Rasipuram, Namakkal DT. Tamilnadu, India for executing this work.

\section{Funding}

Tamilnadu State Council for Science \& Technology (TNSCST), DOTE Campus, Chennai (Science \& Technology Project: TNSCST/STP-PRG/AR/2018-2019).

\section{Conflicts of interest/Competing interests}

There is no conflict of interest.

\section{Availability of data and material}

Not applicable.

Code availability (software application or custom code)

Not applicable.

\section{Authors' contributions}

Sharmila Baburam ${ }^{1}$, Executed the research work.

${ }^{*}$ Gnanendra Shanmugam ${ }^{3}$ and Srinivasan Ramasamy ${ }^{2}$ - performed the methodology

*Maghimaa Mathanmohun ${ }^{1}$-Supervised the work

\section{Ethics approval}

Not applicable.

\section{Consent to participate}

Not applicable

\section{Consent for publication}

Not applicable.

\section{References}

1. Brusselaers, N., Vogelaers, D., \& Blot, S. (2011). The rising problem of antimicrobial resistance in the intensive care unit. Annals of Intensive Care, 1, 47 
2. Najar, M. S., Saldanha, C. L., \& Banday, K. A. (2009). Approach to urinary tract infections. Indian Journal of Nephrology, 19(4), 129-139

3. Ventola, C. L. (2015). The Antibiotic Resistance Crisis: Part 1: Causes and Threats. Pharmacy and Therapeutics, 40(4), 277-283

4. Kabeerdass, V. R., Ramasam, N. S. R. S. G., \& Mathanmohun, M. S., \& (2021). Invitro and insilico studies on antibacterial potentials of phytochemical extracts. Materials Today: Proceedings. https://doi.org/10.1016/j.matpr.2021.05.017

5. Choudhary, K., Singh, M., \& Pillai, U. (2008). Ethnobotanical Survey of Rajasthan - An Update. American-Eurasian Journal of Botany, Vol.1(2), 38-45

6. El-Mahmood Muhammad Abubakar. (2010). Antibacterial potential of crude leaf extracts of Eucalyptus camaldulensis against some pathogenic bacteria. African Journal of Plant Science, Vol. 4(6), 202-209

7. Gnanendra, T. S., Geethu, G., Arjunan, S., Sharmila, B., Zahir Hussain, M. I., Moorthy, K., \& Jeyakumar, N. (2010). Theoretical Models of Quorum Sensing Dependent Regulation of Transcriptional Activators of Pseudomonas aeruginosa. International Journal of Biological Technology, 1, 43-49

8. Gnanendra Shanmugam, Mohamed, S., \& Natarajan, J. (2013). Identification of potent inhibitors for Salmonella typhimurium quorum sensing via virtual screening and pharmacophore modeling. Combinatorial Chemistry \& High Throughput Screening, 16, 826-83914

9. Thompson, J. D., Higgins, D. G., \& Gibson, T. J. (1994). Clustal W: improving the sensitivity of progressive multiple sequence alignment through sequence weighting position- specific gap penalties and weight matrix choice. Nucl Acids Res, 22, 4673-4680

10. Stierand, K., Maab, P., \& Rarey, M. (2006). Molecular Complexes at a Glance: Automated Generation of two-dimensional Complex Diagrams. Bioinformatics, 22, 1710-1716

11. Rarey, M., Kramer, B., Lengauer, T., \& Klebe, G. (1996). A fast flexible docking method using an incremental construction algorithm. J Mol Biol, 261, 470-489

12. Kyoko Kanamaru, K., Kanamaru, Tatsuno, T., Tobe, C., \& Sasakawa (2000). SdiA, an Escherichia coli homologue of quorum-sensing regulators, controls the expression of virulence factors in enterohaemorrhagic Escherichia coli 0157:H7, Molecular Microbiology, 38:805-816.

13. Tavío, M. M., Aquili, V. D., Poveda, J. B., Antunes, N. T., Sánchez-Céspedes, J., \& Vila, J. (2010). Quorum-sensing regulator sdiA and marA overexpression is involved in in vitro-selected multidrug resistance of Escherichia coli. J Antimicrob Chemother, 65, 1178-1186

\section{Figures}



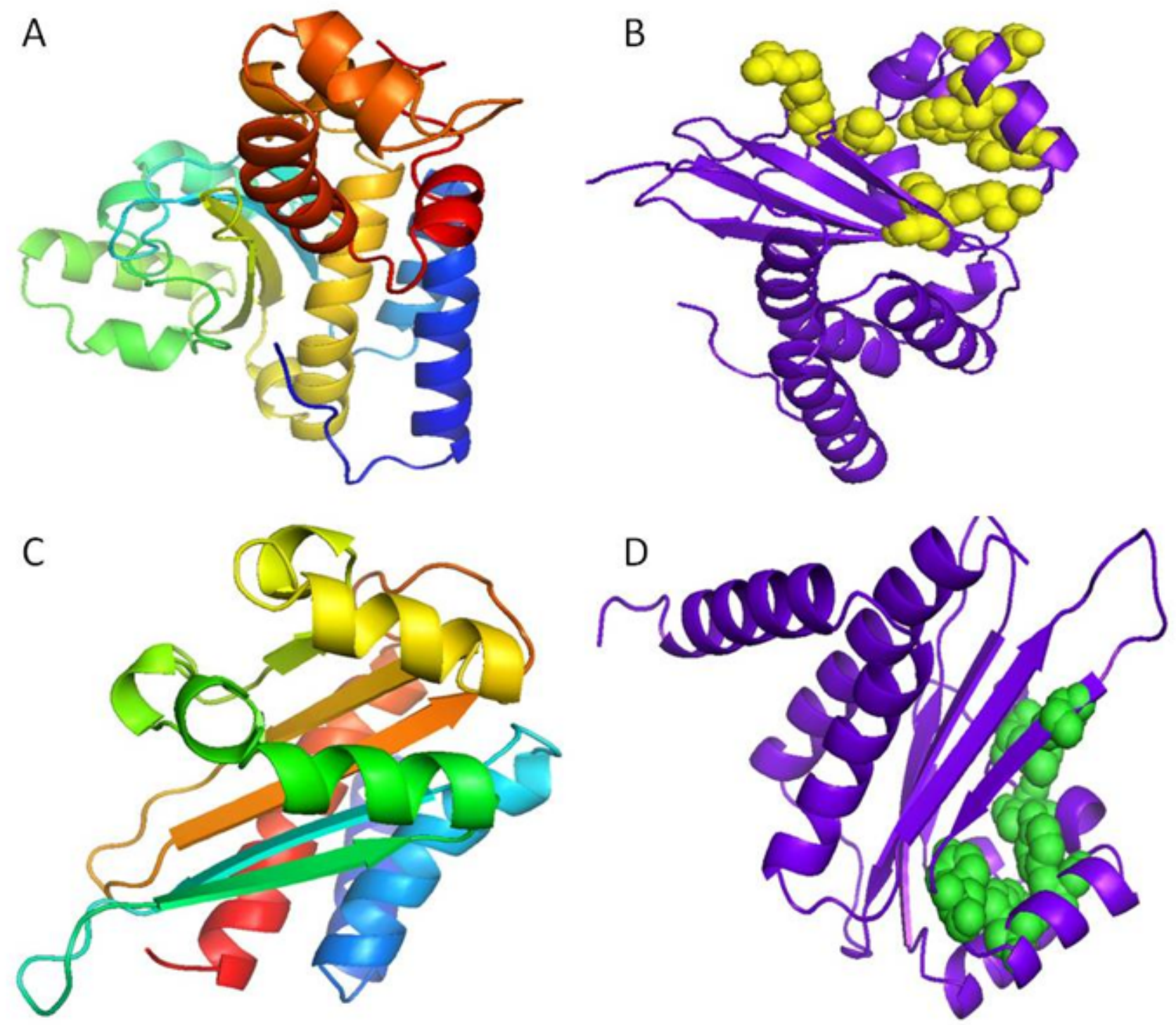

Figure 1: 3D Structure of quorum-sensing transcriptional activators shown in cartoons representation and the active site is highlighted as spheres. A. CviR Protein from Chromobacterium violaceum B. Active site of CviR Protein from Chromobacterium violaceum C. LasR Protein from Pseudomonas aeruginosa D. Active site of LasR Protein from Pseudomonas aeruginosa

Figure 1

See image above for figure legend. 
Sp:P25084|LASR_PSEAE/1-239

triD3W065[D3W065_CHRV/2-265

SP|P25084|LASR_PSEAE/1-239

triD3W065[D3W065_CHRVL/1-265

SP|P25084|LASR_PSEAE/1-239

tr[D3W065[D3W065_CHRVL/1-265

SP/P25084|LASR_PSEAE/1-239

triD3W065[D3W065_CHRV/1-265

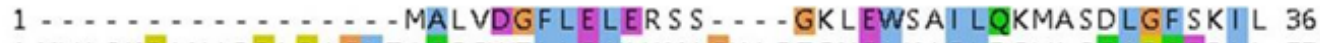
1 MVI SKP I NARPLPAGLTASQQWTLLEWI HMAG̈HI ETENELKAFLDQVLSQAP SERLL 57 37 FGLL - - PKDSQDYENAFI VGNYPAAWR EHYDRAGYARVDPTVSHCTQSVLPI FWEP 90 58 LALGRLNNQNQ I QRLERVLNVSYP SDWLDQYMKENYAQHDP I LR - I HLGQGPVMNEE 113 * * * *

91 SI YQTR - KQHEFFEEASAAGLVYGL TMP LHGARGELG-AL SLSVEAENRAEANRFM 144 114 RFNRAKGAEEKRFI AEATQNGMGSG I TFSAASERNNI GSI L S I AG - . - . REPGRN 164 145 ESVLPT LMMLKDYALQSGAGLAFEHPVSK-PVVLTSREKEVLQWCAI GKT SWEI SVI 200 165 AALVAMLNCLTPHLHQAAI RVANLPPASP SNMPL SQREYDI FHWMSRGKTNWE I ATI 221

Figure 2: The pair-wise sequence alignment between Pseudomonas aeruginosa and Chromobacterium violaceum. The conserved regions were shown in clustal $\mathrm{X}$ color format and the conserved active site is highlighted with rectangle box and also marked with Astrik $(*)$

\section{Figure 2}

See image above for figure legend. 

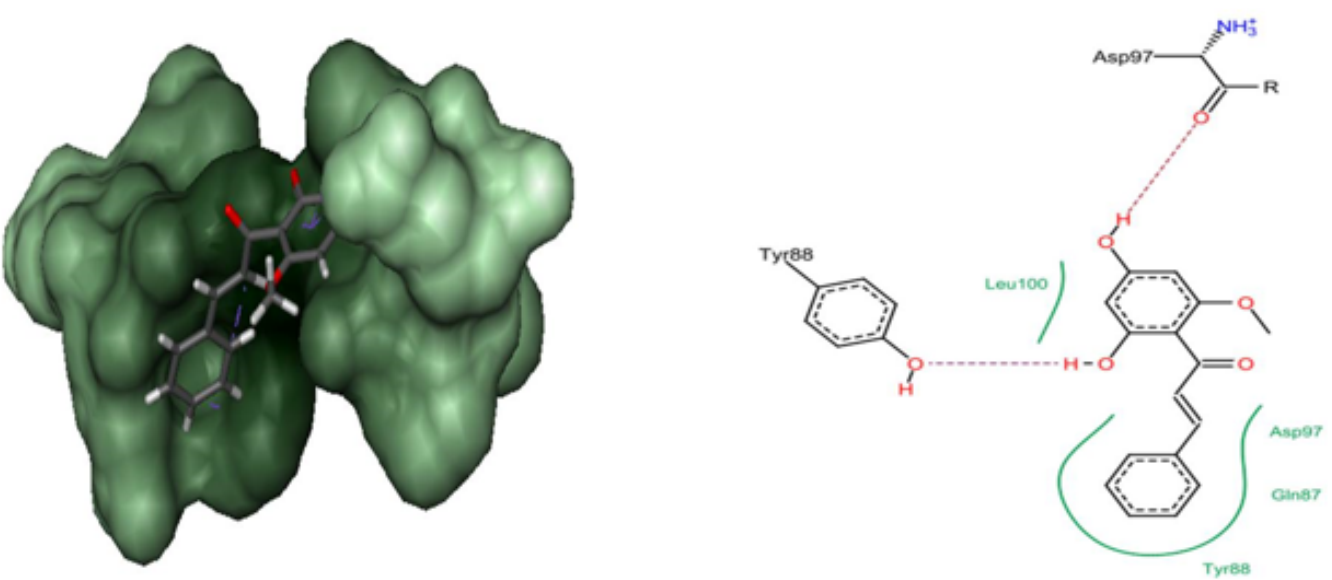

Docking complex and interaction of CID_641785
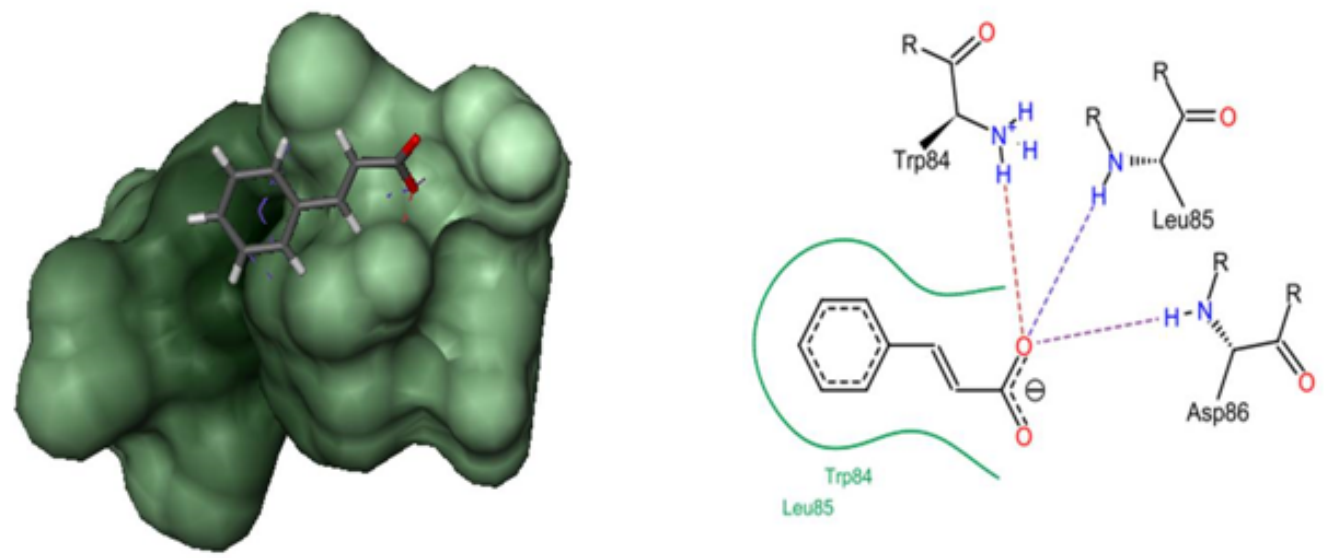

Docking complex and interaction of CID_444539
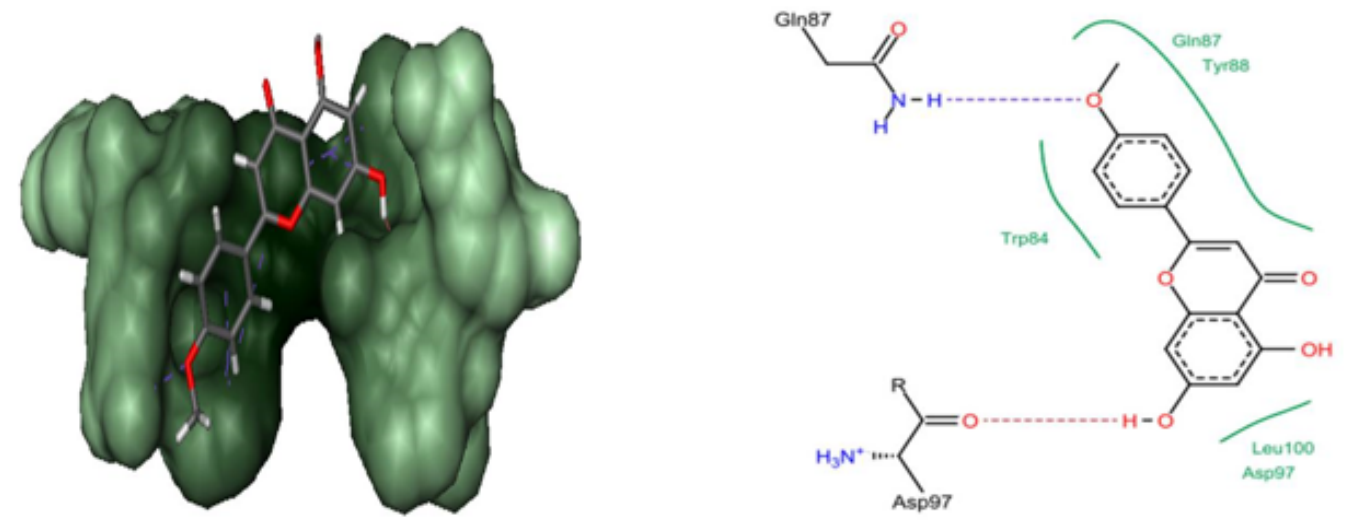

Docking complex and interaction of CID_5280442

\section{Figure 3}

Docking complex and the interactions of the best three compounds with the active site amino acids of CviR from Chromobacterium Violaceum 

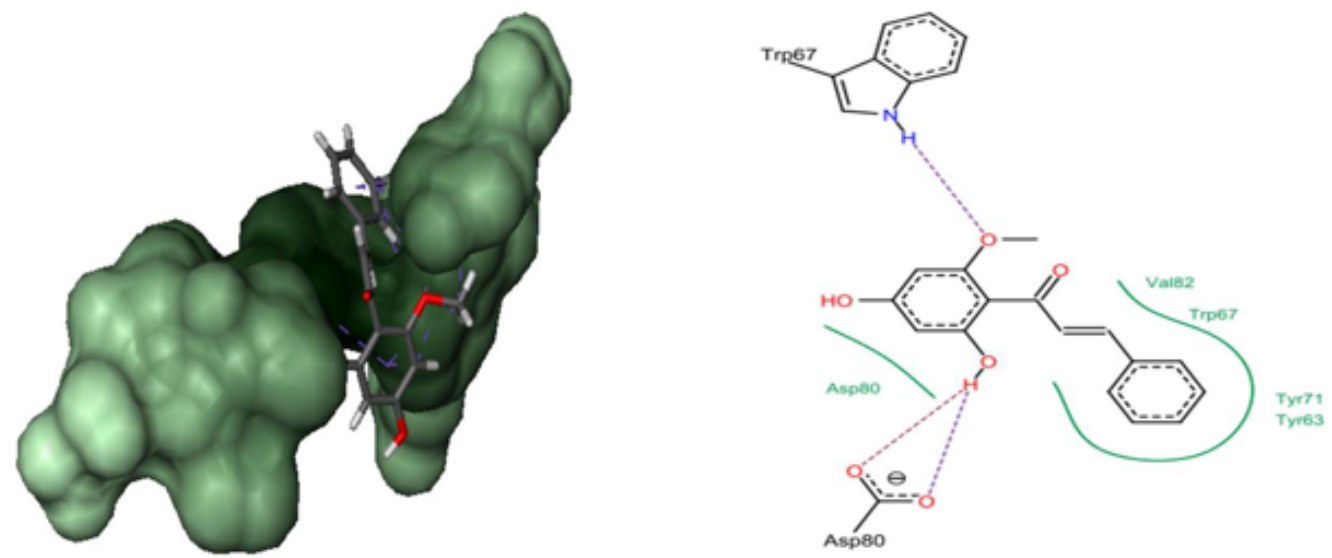

Docking complex and interaction of CID_641785
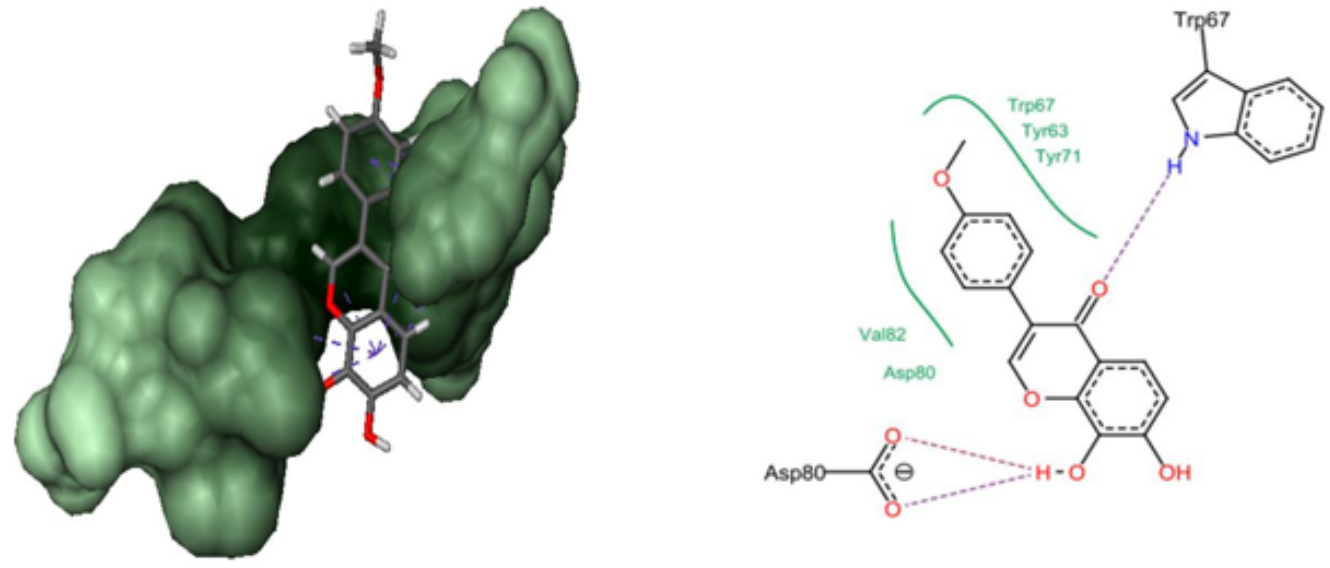

Docking complex and interaction of CID_5481240
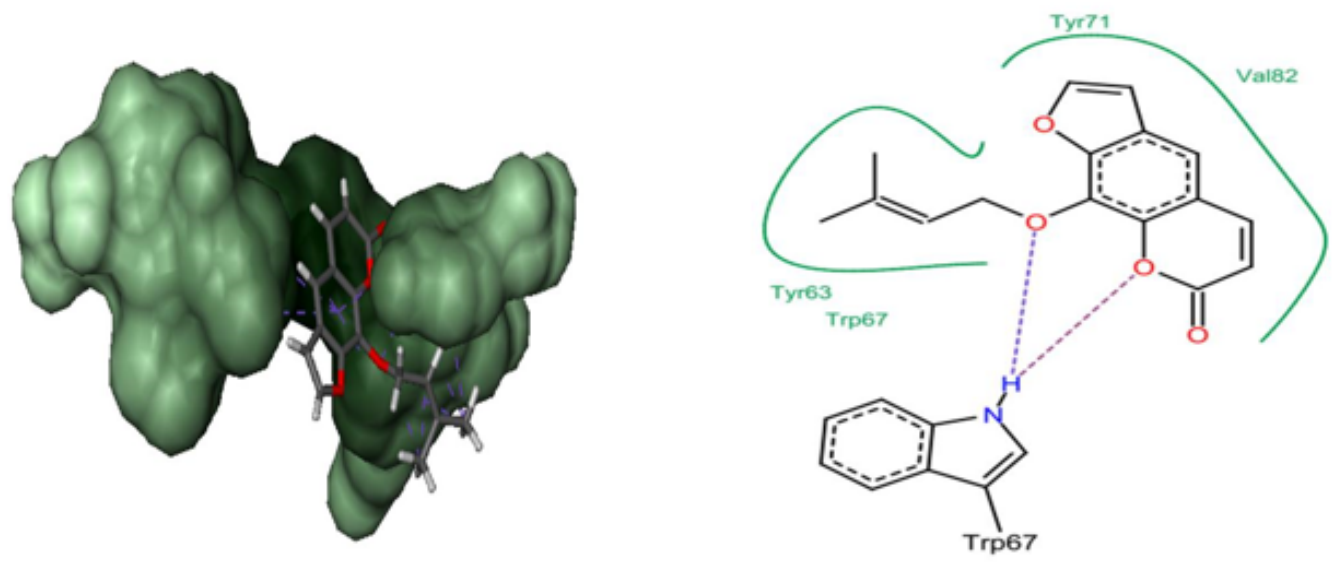

Docking complex and interaction of CID_10212

\section{Figure 4}

Docking complex and the interactions of the best three compounds with the active site amino acids of LasR from Pseudomonas aeruginosa 